\section{A Fast and Efficient User Pairing and Power Allocation Algorithm for Non-orthogonal Multiple Access in Cellular Networks}

\section{J. He, Z. Tang and Z. Che}

Non-orthogonal multiple access (NOMA) is emerging as a promising multiple access technology for the 5th generation cellular networks to address the fast growing mobile data traffic. It applies superposition coding in transmitters, allowing simultaneous allocation of the same frequency resource to multiple intra-cell users. Successive interference cancellation is used at the receivers to cancel intra-cell interference. User pairing and power allocation (UPPA) is a key design aspect of NOMA. Existing UPPA algorithms are mainly based on exhaustive search method with extensive computation complexity, which can severely affect the NOMA performance. In this paper we propose a fast proportional fairness (PF) scheduling based UPPA algorithm to address the problem. The novel idea is to form user pairs around the users with the highest PF metrics with pre-configured fixed power allocation. System level simulation results show the proposed algorithm is significantly faster ( 7 times faster for the scenario with 20 users) with a negligible throughput loss than the existing exhaustive search algorithm.

Introduction: Recently an exponential growth of mobile devices was witnessed with more than half a billion mobile devices (mainly smartphones) added in 2015 [1]. In addition it is expected there are 50 billions Internet of Things (IoT) devices connected to Internet by 2020. The ever-increasing smart mobile devices and IoT devices are driving a rapid growth of mobile data traffic. Significant innovations from cellular networks are needed to address the huge traffic, e.g., ultra-dense small cells, higher order sectorization, massive MIMO, cloud RAN and millimeter wave technologies [2].

Multiple access is a key design aspect of cellular networks. Orthogonal multiple access (OMA) has been used in the first to the 4th generation cellular networks. To achieve higher spectrum efficiency non-orthogonal multiple access (NOMA) is considered as a promising multiple access technology for the 5th generation cellular networks. The root of NOMA is the superposition coding, which allows simultaneous allocation of the same frequency resource to multiple users. Then successive interference cancellation is used at the receivers to cancel intra-cell interference. Research on the application and evaluation of NOMA to the downlink communications of cellular networks has been conducted, reporting around $20 \%$ throughput gain over OMA [3, 4, 5].

In this paper we focus on the problem of user pairing and power allocation (UPPA) for NOMA in downlink communications, which determines the set of intra-cell users multiplexed over the same frequency resource blocks and the power allocation to these users. UPPA is a key decision to make for NOMA with a significant impact on NOMA performance in terms of spectrum efficiency and computational complexity. However, existing UPPA algorithms are mainly based on exhaustive search to find proper user pairs and power allocation, which can result in excessive scheduling processing delay and lead to large system performance loss.

We propose a fast and efficient proportional fairness (PF) scheduling based UPPA algorithm to address the problem. It uses a novel idea of forming user pairs around the users with the highest PF metrics and using pre-configured fixed power allocation over the resource blocks. System level simulator is developed to check the feasibility of the idea and evaluate the proposed UPPA algorithm. Simulation results show that the proposed algorithm is significantly faster than the existing exhaustive search algorithm with negligible throughput loss.

System Model: Let us consider a cellular network with $N_{\text {site }}$ sites equipped with one eNB each. eNB 1 is located in the center of the network. Each eNB has 3 sectors. The $j$ th sector of the $i$ th site is denoted by $\mathcal{A}_{i, j}$, where $i \in\left[1, N_{\text {site }}\right]$ and $j \in[1,3]$.

Without loss of generality we can focus our analysis on the sector $\mathcal{A}_{1,1}$. Suppose we have $N_{\text {ue }}$ users randomly and uniformly distributed in the sector. We also consider a fully loaded network, in which the frequency resources allocated to each sector are fully used. We assume there are $N_{\text {rb }}$ physical resource block (PRB), which represents the basic time-frequency resource unit for data transmission in LTE networks.
Let us now define the signal power $\mathcal{P}_{i, j, u, r}$ received by a general user $u$ from a general sector $\mathcal{A}_{i, j}$ over PRB $r$ as:

$$
\mathcal{P}_{i, j, u, r}=P_{\mathrm{t}} G_{\mathrm{PL}}(i, u) G_{\mathrm{A}}(i, j, u) \psi_{i, u} \phi_{i, j, u, r},
$$

where $P_{\mathrm{t}}$ denotes eNB transmit power over a PRB, $G_{\mathrm{PL}}(i, u)$ denotes the path gain between eNB $i$ and user $u, G_{\mathrm{A}}(i, j, u)$ is antenna gain between sector $\mathcal{A}_{i, j}$ and user $u, \psi_{i, u}$ denotes shadow fading between eNB $i$ and user $u$, and $\phi_{i, j, u, r}$ denotes small scale fast fading between sector $\mathcal{A}_{i, j}$ and user $u$ over PRB $r$. We assume equal and constant transmit power over PRBs and fully correlated shadowing of sectors of a site.

NOMA is designed on top of OFDMA in downlink communications. If a PRB (say $r$ ) is allocated to only one user (say $u$ ), the user receives no intra-cell interference, which is the same for OMA. In this case, let $\gamma_{u, r}^{\mathrm{s}}$ denote the signal to interference plus noise ratio (SINR) of user $u$ over PRB $r$ with superscript s designating OMA, which is computed as:

$$
\gamma_{u, r}^{\mathrm{s}}=\frac{\mathcal{P}_{1,1, u, r}}{\sum_{j=2}^{3} \mathcal{P}_{1, j, u, r}+\sum_{i=2}^{N_{\text {site }}} \sum_{j=1}^{3} \mathcal{P}_{i, j, u, r}} .
$$

It is noted that as the downlink communication is interference limited, noise power is negligible and is not considered in this paper.

If PRB $r$ is allocated to two users (say $u_{1}$ and $u_{2}$ ), without loss of generality, we assume $\gamma_{u_{1}, r}^{s}>\gamma_{u_{2}, r}^{s}$. According to the NOMA principle, at the transmitter side, the desired signals targeting to $u_{1}$ and $u_{2}$ are superimposed over PRB $r$, with transmit power $\alpha P_{\mathrm{t}}$ and $(1-\alpha) P_{\mathrm{t}}$ for users $u_{1}$ and $u_{2}$, respectively. Power allocation coefficient $\alpha$ satisfies the condition of $\alpha<0.5$, otherwise SIC at $u_{1}$ will fail.

At the receiver side, user $u_{2}$ decodes its signal directly without SIC, by which $u_{1}$ signal is treated as intra-cell user interference. Let $\gamma_{u_{1}, r}^{\mathrm{m}}\left(u_{1}, u_{2}, \alpha\right)$ and $\gamma_{u_{2}, r}^{\mathrm{m}}\left(u_{1}, u_{2}, \alpha\right)$ denote SINR of users $u_{1}$ and $u_{2}$ which are multiplexed over PRB $r$ with power allocation ratio $\alpha$, respectively. The superscript $\mathrm{m}$ is designated intra-cell user multiplexing. We can compute $\gamma_{u_{2}, r}^{m}$ by:

$\gamma_{u_{2}, r}^{\mathrm{m}}\left(u_{1}, u_{2}, \alpha\right)=\frac{(1-\alpha) \mathcal{P}_{1,1, u_{2}, r}}{\sum_{j=2}^{3} \mathcal{P}_{1, j, u_{2}, r}+\sum_{i=2}^{N_{\text {site }}} \sum_{j=1}^{3} \mathcal{P}_{i, j, u_{2}, r}+\alpha \mathcal{P}_{1,1, u_{2}, r}}$

At user $u_{1}$ the intra-user interference from $u_{2}$ is decoded first and cancelled before user $u_{1}$ desired signal is decoded. SINR of user $u_{1}$ can be computed by:

$$
\gamma_{u_{1}, r}^{\mathrm{m}}\left(u_{1}, u_{2}, \alpha\right)=\frac{\alpha \mathcal{P}_{1,1, u_{1}, r}}{\sum_{j=2}^{3} \mathcal{P}_{1, j, u_{1}, r}+\sum_{i=2}^{N_{\text {site }}} \sum_{j=1}^{3} \mathcal{P}_{i, j, u_{1}, r}} .
$$

Algorithm Design: So far the SINR model for NOMA with two users sharing one PRB is described. However, from the system point of view, the eNBs need to determine which users to use the PRBs and if they are multiplexed over the same PRBs, and determine the power allocation coefficient $\alpha$. For simplicity, in this paper the number of users that can be multiplexed over a PRB is limited to 2. The exhaustive search (ES) algorithm attempts to find the best user pair and power allocation policy which maximizes the scheduling metric by searching over all the user pairing and power allocation options. The method requires intensive computation. It has a complexity of $\mathcal{O}\left(N_{\mathrm{ue}}^{2} N_{\mathrm{rb}}\right)$, with $N_{\text {ue }}^{2}$ user pairing options over each PRB, which can lead to excessive scheduling delay with a large number of users.

In this paper we propose a fast UPPA algorithm aiming to significantly reduce the computation time. Let $\eta_{u_{1}, r, n}\left(u_{2}, \alpha\right)$ denote the estimated instantaneous achievable throughput of user $u_{1}$ over PRB $r$ at the $n$th subframe, in the case of $u_{1}$ and $u_{2}$ being multiplexed with a ratio $\alpha$ of transmit power allocated to the user with higher SINR. Let $\bar{\eta}_{u, n}$ denotes the average throughput of user $u$ until subframe $n$. For simplicity Shannon formula is applied to compute channel capacity from SINR.

According to the PF scheduling algorithm, a PF metric for a user $u_{1}$ over PRB $r$ shared by $u_{1}$ and $u_{2}$, which is denoted by $F_{u_{1}, r, n}\left(u_{2}, \alpha\right)$, can be computed by:

$$
F_{u_{1}, r, n}\left(u_{2}, \alpha\right)=\frac{\eta_{u_{1}, r, n}\left(u_{2}, \alpha\right)}{\bar{\eta}_{u_{1}, n}} .
$$

Let $F_{u, r, n}$ denote the PF metric for user $u$ over PRB $r$ without intra-cell user multiplexing, which corresponds to the PF metric with OMA. $F_{u, r, n}$ represents a specific case of $F_{u_{1}, r, n}\left(u_{2}, \alpha\right)$, with $u_{1}=u_{2}=u$ and $\alpha=0$. 
The propose UPPA algorithm has two main operation loops for scheduling at each subframe (say $n$th subframe). In the outer loop the user and the PRB with maximal $F_{u, r, n}$ over the unallocated PRBs are identified. Then in the inner loop the second user to be multiplexed with the first user over the PRB identified in the outer loop is determined, which should maximizes the sum of PF metrics of the paired users. In addition the power allocation ratio $\alpha$ is set to 0.2 if two different users are multiplexed. In this way exhaustive search of user pairs and power allocation ratios is avoided. It is noted the first user in the outer loop may have lower SINR than the second user. The proposed UPPA algorithm has a complexity of $\mathcal{O}\left(N_{\mathrm{ue}} N_{\mathrm{rb}}\right)$. The complete algorithm is presented below. The proposed fast UPPA Algorithm

1) Compute SINR $\gamma_{u, r}^{\mathrm{s}}$ and $\eta_{u, r, n}$ at subframe $n$ for all users and PRBs.

2) Compute PF metrics $F_{u, r, n}$ for all users over all PRBs at subframe $n$.

3) Find user $u_{1}^{*}$ and PRB $r^{*}$ which maximizes $F_{u_{1}, r, n}$ over users $u_{1} \in$ $\left[1, N_{\mathrm{ue}}\right]$ and unallocated PRBs (initially including all PRBs).

- For each candidate user $u_{2}$ to be multiplexed with $u_{1}^{*}, u_{2} \in\left[1, N_{\mathrm{ue}}\right]$, compute $\gamma_{u_{1}^{*}, r}^{\mathrm{m}}\left(u_{1}^{*}, u_{2}, \alpha\right)$ and $\gamma_{u_{2}, r}^{\mathrm{m}}\left(u_{1}^{*}, u_{2}, \alpha\right), F_{u_{1}^{*}, r, n}\left(u_{2}, \alpha\right)$ and $F_{u_{2}, r, n}\left(u_{1}^{*}, \alpha\right)$, with $\alpha=0$ if $u_{2}=u_{1}^{*}$ and $\alpha=0.2$ if $u_{2} \neq u_{1}^{*}$.

- Find user $u_{2}^{*}$, which maximizes $F_{u_{1}^{*}, r, n}\left(u_{2}, \alpha\right)+F_{u_{2}, r, n}\left(u_{1}^{*}, \alpha\right)$.

- Allocate PRB $r^{*}$ to user $u_{1}^{*}$ and $u_{2}^{*}$. Mark PRB $r^{*}$ as allocated.

- Update average throughput $\bar{\eta}_{u_{1}^{*}, n}$ and $\bar{\eta}_{u_{2}^{*}, n}$ of users $u_{1}^{*}$ and $u_{2}^{*}$.

- Update the PF metric of users $u_{1}^{*}$ and $u_{2}^{*}$ over unallocated PRBs.

4) Go back to Step 3) until all PRBs are allocated.

Experiment Setup and Simulation Results: A cellular network with 19 eNBs and hexagonal cell layout is simulated. Inter-site distance $R$ is 500 meters. The total transmit power over all PRBs (equal to $N_{\mathrm{rb}} P_{\mathrm{t}}$ ) is set to 24 watts. The model specified in [6] for outdoor line-of-sight communications is used for path loss $G_{\mathrm{PL}}(d)$ :

$$
G_{\mathrm{PL}}(d)=-34.02-22 \log _{10}(d)[\mathrm{dB}] .
$$

where $d$ is the distance between site $i$ and user $u$. Sector antenna model specified in [6] is used with maximum antenna gain of $15.5 \mathrm{dBi}$ and antenna front to back ratio of $25 \mathrm{~dB}$ [2]. Shadow fading $\psi_{i, u}$ between any eNB $i$ and user $u$ is assumed to follow a log-normal distribution with zero mean and $8 \mathrm{~dB}$ standard deviation [6]. The inter-site shadow fading correlation is 0.5 . The small scale fast fading coefficient follows exponential distribution with mean of 1 .

Table 1: Modes of setting on the number of UEs and PRBs.

\begin{tabular}{l|lllll}
\hline Modes & 1 & 2 & 3 & 4 & 5 \\
\hline \# of UEs & 2 & 5 & 10 & 15 & 20 \\
\hline \# of PRBs & 1 & 2 & 3 & 4 & 5 \\
\hline
\end{tabular}

We consider 5 modes of setting on the number of users (or UEs) and PRBs as shown in Table.1. Each PRB has $180 \mathrm{kHz}$. For each setting mode we perform 20 drops of UEs to the network and run 5000 simulation snapshots for each UE drop to obtain mean network performance. In the experiments we compare the proposed fast algorithm to the ES algorithm with PF scheduler. For the ES algorithm all possible user pairings are tested but the power allocation $\alpha$ is fixed to 0.2 as it was found to be a good setting for time saving. It is noted that several variants of the ES algorithm have been proposed [4], but they still have very high computation complexity, which are not compared here due to space limit.

Site throughput and simulation time for networks with PF scheduler and the proposed UPPA algorithm (labelled 'PF-NOMA-Fast') and the ES algorithm (labelled 'PF-NOMA-ES') are compared in Fig. 1 and Fig. 2, respectively. The average throughput computing window for PF scheduler is 20. Results of OMA with round robin (RR) scheduler, NOMA with ES and RR scheduler, and OMA with PF scheduler are also included, labelled 'RR-OMA', 'RR-NOMA-ES' and 'PF-OMA', respectively. For the RR scheduler a number of users $\left(N_{\mathrm{rb}}\right.$ for OMA and $2 N_{\mathrm{rb}}$ for NOMA) are scheduled in round robin order over available PRBs.

According to simulation results, we have the following observations:

- The proposed UPPA algorithm is significantly faster than the ES algorithm. In the scenario with $20 \mathrm{UE}$ and 5 PRBs (setting mode 5), Configuration PF-NOMA-ES uses 1530 seconds, which is 8 times of PF-NOMA-Fast (190 seconds). Meanwhile PF-NOMA-Fast has almost identical site throughput to PF-NOMA-ES.

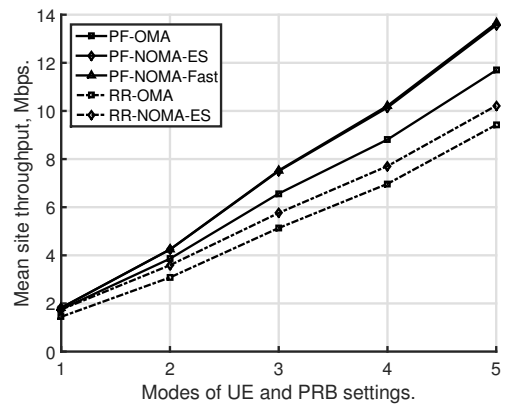

Fig. 1. Mean site throughput (Mbps) versus UE and PRB settings in Table.1.

- Simulation time of PF-NOMA-ES increases fast with squared number of users, while PF-NOMA-Fast and RR scheduler with OMA and NOMA increase much slower.

- NOMA with PF scheduler has obvious throughput gains over OMA with PF scheduler due to user multiplexing over the same PRB (e.g., $16 \%$ gain for UE and RB setting mode 5). And PF scheduler has further large throughput gains over RR scheduler with both OMA and NOMA due to better exploitation of user diversity.

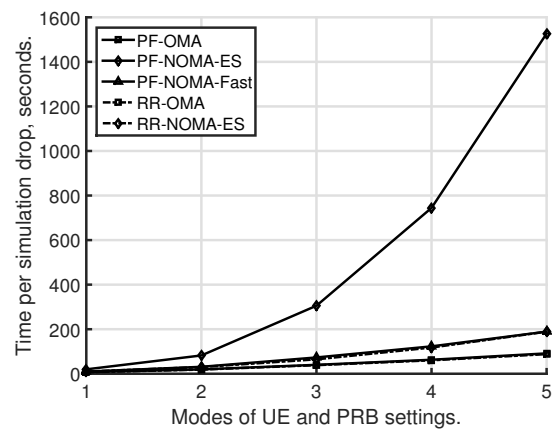

Fig. 2. Simulation time per drop (seconds) versus UE and PRB settings.

Conclusion: We proposed a fast and simple user pairing algorithm which forms user pairs around the users with the highest PF metrics and uses a pre-configured ratio for power allocation. Simulations showed that the proposed algorithm is significantly faster than the exhaustive search algorithm with negligible throughput loss. Simulations also confirmed large throughput gains of NOMA over OMA and of PF scheduler over RR scheduler. The proposed algorithm can be applied to other antenna technologies and channel models, which is left for our future works.

Acknowledgment: The authors would like to acknowledge the financial support of project DETERMINE (FP7-PEOPLE-2012IRSES Proposal number: 318906)

Jianhua He and Zuoyin Tang (Aston University, School of Engineering and Applied Science, Birmingham, B4 7ET, UK)

Zhongzhi Che (Yantai Institute of China Agricultural University, 264670, Yantai China), Corresponding Author.

E-mail: 326896856@qq.com

\section{References}

1 Cisco, 'Cisco Visual Networking Index: global mobile data traffic forecast update, 2015 - 2020', White Paper, 2016.

2 J. He et al, 'Analytical evaluation of higher order sectorization, frequency reuse, and user classification methods in OFDMA networks', IEEE Trans. Wireless Comm., Sept. 2016. DOI: 10.1109/TWC.2016.2613515.

3 Y. Saito et al, 'Non-orthogonal multiple access (NOMA) for cellular future radio access', IEEE VTC Spring'13, June 2013.

4 A. Benjebbour et al, 'System-level performance of downlink NOMA for future LET enhancements', Globecom Workshops'13, Dec. 2013.

5 Z. Ding et al, 'The application of MIMO to Non-orthogonal multiple access', IEEE Trans. Wireless Comm., Jan. 2016, 15, p.537-552.

6 3GPP TR 36.814 V9.0.0, 'Further advancements for E-UTRA physical layer aspects', Technical Report, March 2010. 